\title{
A NEW METHOD FOR ONE-POT SYNTHESIS OF ARYLOXYPHENOXYPROPIONATE HERBICIDES USING 2,4,6-TRICHLORO-1,3,5-TRIAZINE AND $\left({ }^{n} \text {-BU }\right)_{4}$ NI AS A HOMOGENEOUS CATALYST
}

\author{
MEHDI KALHOR ${ }^{A *}$, AKBAR DADRAS ${ }^{*}$, AKBAR MOBINIKHALEDI ${ }^{C}, H A S S A N ~ T A J I K{ }^{D}$ \\ ${ }^{a}$ Department of Chemistry, Payame Noor University, Tehran 19395-4697, I. R. of Iran \\ ${ }^{b}$ Department of Chemistry, Islamic Azad University, East Tehran Branch, 33955-163, Qiamdasht, Iran \\ ${ }^{c}$ Department of Chemistry, Arak University, Arak, Iran \\ ${ }^{d}$ Department of Chemistry, Guilan University, Rasht, Iran, 41335-1914 \\ (Received: September 15, 2010 - Accepted: June 20, 2011)
}

\begin{abstract}
The one-pot reaction of halo-heterocycle, (R)-4-hydroxyphenoxy propionic acid and an alcohol, amine or sulfonamide is described as an efficient method for the synthesis of aryloxyphenoxy propionate hrerbicides by using 2,4,6-trichloro-1,3,5-triazine in the presence of $\left({ }^{n} \text {-Bu }\right)_{4} \mathrm{NI}$, as a homogeneous catalyst under mild conditions. The present procedure offers several advantages, such as good yields, short reaction times and easy workup.
\end{abstract}

Keywords: One-pot reaction, Catalyst, Aryloxyphenoxy propionate, Cyanuric chloride

\section{INTRODUCTION}

The one-pot reaction is known as a reaction in which three or more easily accessible compounds are combined in a single reaction vessel. ${ }^{1-6}$ One-pot reactions increase the efficiency of reactants by combing several operational steps without isolation of intermediates or changing the reaction conditions. Speed, diversity, efficiency and environmental amiability are some of the major advantages of these reactions. They have emerged as valuable tools for the preparation of structurally diverse chemical libraries of drug-like heterocyclic compounds. ${ }^{7-9}$ Furthermore; aryloxyphenoxypropionates (APPs) are a highly effective class of herbicides due to their high activity, high selectivity and low toxicity. Up to now, more than 20 kinds of APPs such as clodinafop-propargyl 1, fenoxaprop-ethyl 2, fluazifop-butyl 3, haloxyfop-methyl 4, haloxyfop-etotyl $\mathbf{5}$, quizalofop-ethyl $\mathbf{6}$, have been commercialized and marketed by major agrochemical companies. ${ }^{10-17}$ They are used effectively in a number of crops including soybeans and cereal grains, such as wheat and rice, to control grass weeds. ${ }^{12}$ Commonly, there are three pathways (route A, B and C) described in the literature for preparation of desired APPs. ${ }^{14-16}$ These routes normally proceed via an aromatic nucleophilic substitution of proper halo-hetero cyclic compounds with (R)-4-hydroxyphenoxy propionic acid (4-HPPA) or it esters (Figure 1).

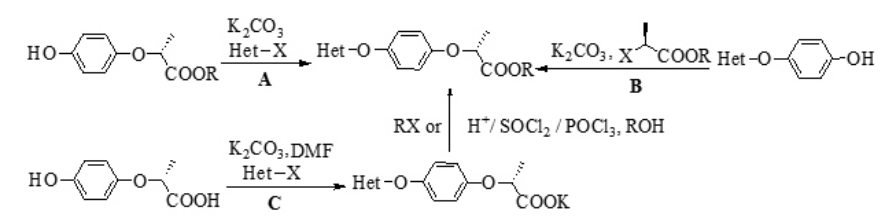

Figure 1. Classical pathways of APPs.

However, due to the low yield and purity of the A and B routes, commonly route $\mathrm{C}$ is chosen as the synthetic strategy for the synthesis of APPs such as clodinafop-propargyl $\mathbf{1}$ from corresponding halo-pyridine, 4-HPPA, and propargyl halides. ${ }^{16}$ Recently, a few efficient examples have been reported for the synthesis of APPs in ionic liquid media. ${ }^{18}$ The routes developed so far suffer from harsh and cumbersome conditions, long reaction times, the use of extremely anhydrous conditions, expensive and toxic reagents such as propargyl halide, the use of large excess of reagents, tedious work-up and by product formation. Because of the commercially importance of APPs, search for the development of a simple, mild, and efficient method is still highly demanded. On the other hand, over the past few years 2,4,6-Trichloro-1,3,5-triazine (cyanuric chloride, CC) has been used in many chemical transformations ${ }^{19}$, especially in conversion of carboxylic acid to ester or amide ${ }^{20-22}$, hence using CC in preparation of APPs would be a challenge for the usual routes (A, B, C) being a valid alternative route.
In view of this report and also due to our attention in one-pot synthesis of organic compounds ${ }^{23,24}$, we are going to describe a practical and more economical method for large-scale preparation of APPs herbicides 1-12.

\section{EXPERIMENTAL}

All used chemicals were purchased from Merck or Fluka Company. Melting points were determined using an electro thermal digital apparatus and are uncorrected. Infrared (IR) spectra were recorded on a Galaxy series Fourier transform infrared (FT-IR) 5000 spectrometer using $\mathrm{KBr}$ discs. ${ }^{1} \mathrm{H}$ NMR and ${ }^{13} \mathrm{C}$ NMR spectra were recorded on Brucker spectrophotometer (300 MHZ) in $\mathrm{CDCl}_{3}$ or DMSO- $d_{6}$ using $\mathrm{Me}_{4} \mathrm{Si}$ as an internal standard. Elemental analyses were performed on a Vario EL III elemental analyzer and mass spectra were recorded on Bruker Biflex Maldi-tof spectrometer.

\section{General one-pot procedure}

To the stirred solution of (R)-2-(4-hydroxyphenoxy)-propionic acid (1.82 $\mathrm{g}, 0.01 \mathrm{~mol})$ in $10 \mathrm{ml}$ of DMF, potassium phosphate $(2.12 \mathrm{~g}, 0.01 \mathrm{~mol})$ was added at $50^{\circ} \mathrm{C}$. Then halo-heterocycle $(0.01 \mathrm{~mol})$ and tetrabutylammonium iodide $(0.036 \mathrm{~g}, 1 \mathrm{~mol} \%)$ was added, stirred at $50-60{ }^{\circ} \mathrm{C}$ for $2 \mathrm{~h}$. After cooling the reaction mixture to $5^{\circ} \mathrm{C}$, cyanuric chloride $(0.738 \mathrm{~g}, 0.004 \mathrm{~mol})$ was added over $10 \mathrm{~min}$, mixed for $1 \mathrm{~h}$ at $25^{\circ} \mathrm{C}$ and subsequently $0.012 \mathrm{~mol}$ alcohol or amine was added. After completion of the reaction 1-2 $\mathrm{h}$ (monitored by thinlayer chromatography, TLC, eluent $n$-hexane: EtOAc $=2: 1$ ), the mixture was poured on $50 \mathrm{~g}$ of crushed ice with stirring and $\mathrm{pH}$ was adjusted to 8 with $25 \%$ $\mathrm{NaOH}$ and stirred for $10 \mathrm{~min}$ at $0-5^{\circ} \mathrm{C}$. The resulting solid was collected by filtration, purified by recrystallization from $90 \%$ ethanol.

Spectroscopic Data for new Compounds:

2-[4-(5-Chloro-3-fluoro-pyridin-2-yloxy)-phenoxy]-propionic acid hydrazide (10); IR (KBr): $v_{\max }=3356,3277(\mathrm{~N}-\mathrm{H}), 1677(\mathrm{C}=\mathrm{O}), 1620,1504$ $(\mathrm{C}=\mathrm{N}), 1453,1239(\mathrm{C}=\mathrm{C}), 1197(\mathrm{C}-\mathrm{O}), 851(\mathrm{C}-\mathrm{Cl}) \mathrm{cm}^{-1} ;{ }^{1} \mathrm{H} \mathrm{NMR}\left(\mathrm{CDCl}_{3}, 300\right.$ MHz): $\delta_{\mathrm{H}} 7.82(1 \mathrm{H}, \mathrm{s}, \mathrm{Ar}), 7.47(1 \mathrm{H}, \mathrm{s}, \mathrm{Ar}), 7.05\left(2 \mathrm{H}, \mathrm{d}, J=8.6 \mathrm{~Hz}, \mathrm{H}_{\mathrm{ph}}\right), 6.89$ $(2 \mathrm{H}, \mathrm{d}, J=8.6 \mathrm{~Hz}, \mathrm{H}), 5.90(1 \mathrm{H}, \mathrm{s}, \mathrm{N}-\mathrm{H}), 5.25(1 \mathrm{H}, \mathrm{q}, J=6.5 \mathrm{~Hz}, \mathrm{CH}), 4.70$ $\left(2 \mathrm{H}, \mathrm{br}, \mathrm{NH}_{2}\right), 1.55\left(3 \mathrm{H}, \mathrm{d}, J=6.5 \mathrm{~Hz}, \mathrm{CH}_{3}\right) \mathrm{ppm} ;{ }^{13} \mathrm{C} \mathrm{NMR}\left(\mathrm{CDCl}_{3}, 75 \mathrm{MHz}\right)$ : $\delta_{C} 18.7,74.1,116.3,122.5,125.0,140.5,145.1,147.2,148.6,151.2,154.1$, $172.19(\mathrm{C}=\mathrm{O}) \mathrm{ppm} ;[\mathrm{M}]^{+} \mathrm{m} / \mathrm{z}=325.72$. Found: MALDI-TOF-MS: $[\mathrm{M}+\mathrm{Na}]^{+}=$ 448.72; Anal Calcd for $\mathrm{C}_{14} \mathrm{H}_{13} \mathrm{ClFN}_{3} \mathrm{O}_{3}: \mathrm{C}, 51.62 ; \mathrm{H}, 4.02 ; \mathrm{N}, 12.90$; Found: $\mathrm{C}$, $51.44 ; \mathrm{H}, 4.03 ; \mathrm{N}, 12.95$.

(R)-2-(4-(5-chloro-3-fluoropyridin-2-yloxy)phenoxy)- $N$-(4,6-dimethoxy pyrimidin- 2-yl) propan amide (11): IR $(\mathrm{KBr}): \mathrm{v}=3409(\mathrm{~N}-\mathrm{H}), 2956(\mathrm{C}-$ $\mathrm{H}), 1719(\mathrm{C}=\mathrm{O}), 1605,1574(\mathrm{C}=\mathrm{N}), 1450(\mathrm{C}=\mathrm{C}), 1166(\mathrm{C}-\mathrm{O}) \mathrm{cm}^{-1} ;{ }^{1} \mathrm{H}$ NMR $\left(\mathrm{CDCl}_{3}, 300 \mathrm{MHz}\right): \delta_{\mathrm{H}} 8.68(1 \mathrm{H}, \mathrm{s}, \mathrm{N}-\mathrm{H}), 7.81(1 \mathrm{H}, \mathrm{s}, \mathrm{Ar}), 7.45(1 \mathrm{H}, \mathrm{s}, \mathrm{Ar})$, $7.09\left(2 \mathrm{H}, \mathrm{d}, J=8.6 \mathrm{~Hz}, \mathrm{H}_{\mathrm{ph}}\right), 6.99\left(2 \mathrm{H}, \mathrm{d}, J=8.6 \mathrm{~Hz}, \mathrm{H}_{\mathrm{ph}}\right), 5.76\left(1 \mathrm{H}, \mathrm{s}, \mathrm{H}_{\text {pyrim }}\right)$, $4.87(1 \mathrm{H}, \mathrm{q}, J=6.5 \mathrm{~Hz}, \mathrm{Ch}), 3.90\left(6 \mathrm{H}, \mathrm{s}, \mathrm{OCH}_{3}\right), 1.65\left(3 \mathrm{H}, \mathrm{d}, J=6.5^{\mathrm{pyrim}} \mathrm{Hz}\right.$, $\left.\mathrm{CH}_{3}\right) \mathrm{ppm} ;{ }^{13} \mathrm{C} \mathrm{NMR}\left(\mathrm{CDCl}_{3}, 75 \mathrm{MHz}\right): \delta_{\mathrm{C}} 18.5,54.2,76.0,85.4,116.7,122.6$, $124.1,140.1,145.1,147.5,148.6,151.1,154.1,155.5,169.9,172.0(\mathrm{C}=\mathrm{O})$ ppm; Calcd: $[\mathrm{M}]^{+} \mathrm{m} / \mathrm{z}=448.83$; Found: MALDI-TOF-MS: $[\mathrm{M}+\mathrm{Na}]^{+}=471.83$; Anal Calcd for $\mathrm{C}_{20} \mathrm{H}_{18} \mathrm{ClFN}_{4} \mathrm{O}_{5}: \mathrm{C}, 53.52 ; \mathrm{H}, 4.04 ; \mathrm{N}, 12.48$. Found: C, 53.71; 
$\mathrm{H}, 4.05 ; \mathrm{N}, 12.48$.

(R)-2-Ethanesulfonyl-imidazo [1,2-a] pyridine-3-sulfonic acid [2-[4-(5-chloro- 3-fluoro-pyridin-2-yloxy)-phenoxy]-propionyl]-amide (12): IR (KBr): $v_{\max }=3275(\mathrm{~N}-\mathrm{H}), 1640(\mathrm{C}=\mathrm{O}), 1605,1457,(\mathrm{C}=\mathrm{N}, \mathrm{C}=\mathrm{C}), 1313$ $(\mathrm{S}=\mathrm{O}), 1167(\mathrm{C}-\mathrm{O}), 735(\mathrm{C}-\mathrm{Cl}) \mathrm{cm}^{-1} ;{ }^{1} \mathrm{H}$ NMR (DMSO-d, $\left.300 \mathrm{MHz}\right): \delta_{\mathrm{H}} 8.97$ $(1 \mathrm{H}, \mathrm{s}, \mathrm{NH}), 8.15\left(1 \mathrm{H}, \mathrm{m}, \mathrm{H}_{\mathrm{imid}}\right), 8.00(1 \mathrm{H}, \mathrm{Ar}), 7.73\left(1 \mathrm{H}, \mathrm{m}, \mathrm{H}_{\mathrm{imid2}}.\right) 7.50$ $(1 \mathrm{H}, \mathrm{Ar}), 7.19\left(2 \mathrm{H}, \mathrm{m}, \mathrm{H}_{\text {imida }}\right), 6.95\left(2 \mathrm{H}, \mathrm{d}, J=8.6 \mathrm{~Hz}, \mathrm{H}_{\mathrm{ph}}\right), 6.60(2 \mathrm{H}, \mathrm{d}, J=$ $8.6 \mathrm{~Hz}, \mathrm{H}), 4.36(1 \mathrm{H}, \mathrm{q}, J=6.5 \mathrm{~Hz}, \mathrm{CH}), 3.66\left(2 \mathrm{H}, \mathrm{m}, \mathrm{CH}_{2}\right), 1.31(3 \mathrm{H}, \mathrm{d}, J=$ $\left.6.5 \mathrm{~Hz}, \mathrm{CH}_{3}\right), 1.13\left(3 \mathrm{H}, \mathrm{m}, \mathrm{CH}_{3}\right) \mathrm{ppm} ;{ }^{13} \mathrm{C}$ NMR (DMSO-d 6 , $\left.75 \mathrm{MHz}\right): \delta_{\mathrm{C}} 7.2$, $19.4,39.1,40.7,48.8,75.9,114.9,115.6,118.5,122.4,124.6,125.9,127.0$, 128.4, 129.1, 140.7, 142.4, 142.7, 145.9, 155.7, $178.0(\mathrm{C}=\mathrm{O}) \mathrm{ppm}$; Calcd: $[\mathrm{M}]^{+}$ $\mathrm{m} / \mathrm{z}=582.04$; Found: MALDI-TOF-MS: $[\mathrm{M}+\mathrm{Na}]^{+}=605.02$; Anal Calcd for $\mathrm{C}_{23} \mathrm{H}_{20} \mathrm{ClFN}_{4} \mathrm{O}_{7} \mathrm{~S}_{2}$ : C, 47.38; H, 3.46; N, 9.61; S, 11.00; Found: C, 47.14; H, $3.45 ; \stackrel{N}{N}, 9.63 ; \mathrm{S}, 11.08$.

\section{RESULTS AND DISCUSSION}

In this work we attempted to synthesize some APPs via a one pot reaction employing $\mathrm{CC} / \mathrm{K}_{3} \mathrm{PO}_{4}$ as a mild and inexpensive reagent in the presence of TBAI as a homogeneous catalyst.

Initially, to evaluate a one-pot process, we prepared ester 1 by reaction between 5-chloro-2,3-difluoro pyridine, (R)-4-hydroxyphenoxy propionic acid and propargyl alcohol using base $/ \mathrm{CC} / 60^{\circ} \mathrm{C}$, these conditions were selected as a model reaction. For optimization, the reaction was carried out with different bases and solvents under the same conditions to increase the product yield. The results are depicted in Table 1. As results showed employing $\mathrm{K}_{3} \mathrm{PO}_{4}$ as base in DMF (Table 1, entry 4) afforded moderate yield of the corresponding ester.

Table 1. The effects of various solvents and base on the one-pot model reaction ${ }^{\mathrm{a}}$.

\begin{tabular}{ccccc}
\hline Entry & Solvent & Base & Total time (h) & Yield $(\%)^{\mathrm{b}}$ \\
\hline 1 & acetone & $\mathrm{K}_{2} \mathrm{CO}_{3}$ & 12 & 38 \\
2 & acetone & $\mathrm{K}_{3} \mathrm{PO}_{4}$ & 12 & 42 \\
3 & $\mathrm{DMF}$ & $\mathrm{K}_{2} \mathrm{CO}_{3}$ & 8 & 52 \\
4 & $\mathrm{DMF}$ & $\mathrm{K}_{3} \mathrm{PO}_{4}$ & 8 & 58 \\
5 & $\mathrm{MeCN}$ & $\mathrm{K}_{3} \mathrm{PO}_{4}$ & 10 & 48 \\
6 & $\mathrm{MeCN}$ & $\mathrm{K}_{3} \mathrm{PO}_{4}$ & 10 & 50 \\
7 & $n$-Hexane & $\mathrm{K}_{3} \mathrm{PO}_{4}$ & 12 & $\mathrm{NR}^{\mathrm{c}}$ \\
8 & $n$-Hexane & $\mathrm{NEt}_{3}$ & 12 & NR \\
9 & $\mathrm{CH}_{2} \mathrm{Cl}_{2}$ & $\mathrm{~K}_{2} \mathrm{CO}_{3}$ & 10 & Trace \\
10 & $\mathrm{CH}_{2} \mathrm{Cl}_{2}$ & $\mathrm{NEt}_{3}$ & 10 & NR \\
\hline
\end{tabular}

${ }^{\text {a }}$ Reaction temperature: $50-60{ }^{\circ} \mathrm{C}$

${ }^{\mathrm{b}}$ Isolated yields

${ }^{\mathrm{c}}$ Not reacted

We also studied the role of various catalysts on the model reaction and the results are summarized in Table 2 . The results show that using $1 \mathrm{~mol} \%$ of $\left({ }^{n} \text {-But }\right)_{4} \mathrm{NI}$ at temperature of $60^{\circ} \mathrm{C}$, in DMF for $4 \mathrm{~h}$ afforded the corresponding product in $89 \%$ yield (Table 2: entry 6). To study the effect of catalyst, the reaction was carried out in absence and presence of catalyst under the same conditions. The reaction product in absence of catalyst, even under longer reaction times was obtained in moderated yield (Table 1: entry 4). It was also found that a higher amount of catalyst did not improve the yield of reaction.
Table 2. The effects of various Catalysts on one-pot model reaction.

\begin{tabular}{ccccccc}
\hline Entry & Catalyst & $\begin{array}{c}\text { Catalyst } \\
\text { load } \\
(\mathrm{mol} \%)\end{array}$ & Solvent & Base & $\begin{array}{c}\text { Total } \\
\text { time }(\mathrm{h})\end{array}$ & $\begin{array}{c}\text { Yield } \\
(\%)\end{array}$ \\
\hline 1 & TBAB $^{\mathrm{a}}$ & 2 & acetone & $\mathrm{K}_{2} \mathrm{CO}_{3}$ & 8 & 58 \\
2 & TBAB & 2 & acetone & $\mathrm{K}_{3} \mathrm{PO}_{4}$ & 8 & 60 \\
3 & TBAI $^{\mathrm{b}}$ & 2 & $\mathrm{ACN}$ & $\mathrm{K}_{2} \mathrm{CO}_{3}$ & 6 & 65 \\
4 & TBAI & 2 & $\mathrm{ACN}$ & $\mathrm{K}_{3} \mathrm{PO}_{4}$ & 6 & 71 \\
5 & TBAI & 2 & $\mathrm{DMF}$ & $\mathrm{K}_{3} \mathrm{PO}_{4}$ & 4 & 85 \\
6 & TBAI & 1 & $\mathrm{DMF}$ & $\mathrm{K}_{3} \mathrm{PO}_{4}$ & 4 & 89 \\
7 & TBAI & 0.5 & $\mathrm{DMF}$ & $\mathrm{K}_{3} \mathrm{PO}_{4}$ & 4 & 70 \\
8 & TBAB $^{2}$ & 2 & $\mathrm{DMF}$ & $\mathrm{K}_{3} \mathrm{PO}_{4}$ & 4 & 84 \\
9 & BTPPB $^{\mathrm{c}}$ & 2 & $\mathrm{DMF}$ & $\mathrm{K}_{3} \mathrm{PO}_{4}$ & 4 & 82 \\
10 & BTEAC $^{\mathrm{d}}$ & 2 & $\mathrm{DMF}$ & $\mathrm{K}_{3} \mathrm{PO}_{4}$ & 4 & 65 \\
11 & HDMSC $^{\mathrm{c}}$ & 2 & $\mathrm{DMF}$ & $\mathrm{K}_{3} \mathrm{PO}_{4}$ & 4 & 74 \\
\hline
\end{tabular}

aTetrabutylammonium bromide, ${ }^{\mathrm{b}}$ Tetrabutylammonium iodide, 'Benzyltriphenyl phosphoniumbromide, ${ }^{d}$ Benzyltriethylammonium chloride, ${ }^{\mathrm{e}}$ Hexadecyl dimethylsulfonium chloride

To examine the generality and efficiency of this simple protocol, we synthesized several APP proven herbicides and three new compounds (the new compounds are very likely to have herbicide activity, but haven't been tested), employing a one-pot reaction of halo-heterocycle, (R)-4-hydroxyphenoxy propionic acid and an alcohol, amine or sulfonamide (Figure 2).<smiles>[R][Y]C(=O)[C@H](C)Oc1ccc(OCC)cc1</smiles>

Figure 2. Synthesis of APP herbicides

As showed in Figure 2, various halo-heterocyclic compounds were reacted efficiently with 4-HPPA in DMF/ $\mathrm{K}_{3} \mathrm{PO}_{4} / \mathrm{TBAI}$ to yield APPA salts, which subsequently underwent insitu esterification, amidation, or sulfonamidation reactions, which proceeded efficiently by using $\mathrm{CC}$. It is noteworthy to mention that the cyanuric acid can be easily recovered at the end of reaction. The results are listed in Table 3. 
Table 3. One-pot synthesis of various APPs, (1-12) using $\mathrm{K}_{3} \mathrm{PO}_{4} / \mathrm{TBAI} / \mathrm{DMF} / \mathrm{CC}$ system

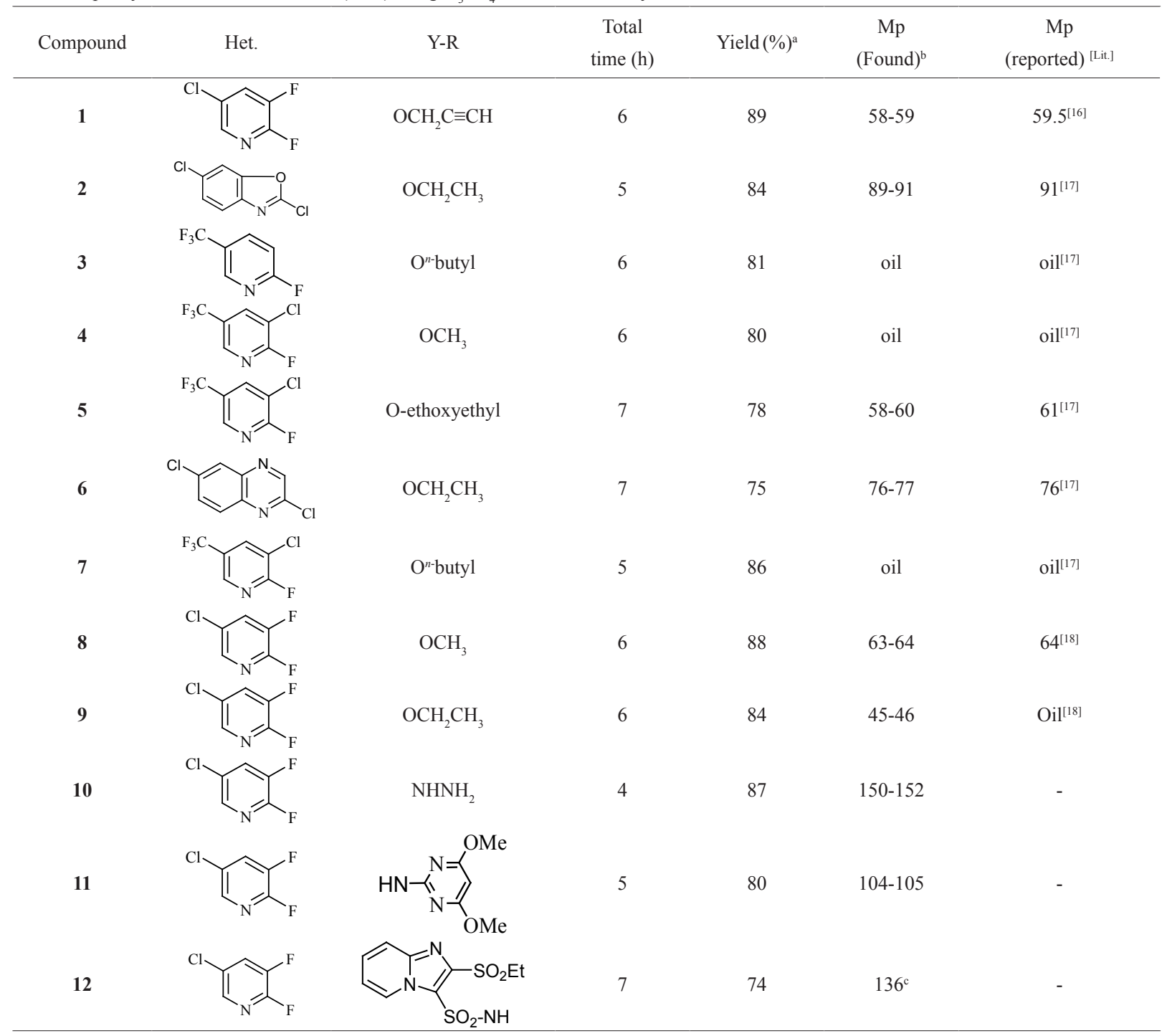

${ }^{a}$ Isolated yields

${ }^{\mathrm{b}}$ Melting points are not corrected.

${ }^{\mathrm{c}}$ Decompose

The structure of new products (10-12) was characterized by the spectroscopic data. The IR, ${ }^{1} \mathrm{H}$ - and ${ }^{13} \mathrm{C}$-NMR spectra of all new synthesized APPs are consistent with their structures. The obtained elemental analysis data are in good agreement with the theoretical values. The IR spectra of $\mathbf{1 0}$ showed an N-H stretching absorption near 3356, $3277 \mathrm{~cm}^{-1}$ and also $\mathrm{C}=\mathrm{O}$ stretching band at $1677 \mathrm{~cm}^{-1}$. Its ${ }^{1} \mathrm{H}-\mathrm{NMR}$ spectra showed a singlet at 5.90 and $4.70 \mathrm{ppm}$ for $\mathrm{CONH}$ and $\mathrm{NH}_{2}$ groups, respectively. The IR and ${ }^{1} \mathrm{H}-\mathrm{NMR}$ spectra of $\mathbf{1 1}$ showed an amidic N-H group in stretching absorption at $3409 \mathrm{~cm}^{-1}$ and 8.68 ppm respectively. The other signals were observed at the expected regions. The ${ }^{13} \mathrm{C}-\mathrm{NMR}$ spectrum of $\mathbf{1 2}$ showed 21 carbon signals and MALDI-TOFMS spectrum revealed $[\mathrm{M}+\mathrm{Na}]^{+}$at 605.02 which elucidate the structure of the reaction product.

\section{CONCLUSION}

In conclusion, by using $\mathrm{K}_{3} \mathrm{PO} / \mathrm{TBAI} / \mathrm{DMF} / \mathrm{CC}$, a convenient general onepot new protocol has been developed to convert various heterocyclic halide/ 4-HPPA/ alcohol, amine or sulfamide directly to the corresponding ester, amide or sulfonamide derivatives under mild condition.

\section{ACKNOWLEDGMENTS}

We wish to express our deep gratitude to the Payame Noor University Research council and also Industrial Pesticide Research Center Karaj for the partial support of the work. 


\section{REFERENCES}

1.- I. Ugi, A. Dömling, W. Hörl, Endeavour 18, 115, (1994).

2.- L.F. Tietze, Chem. Rev. 96, 115, (1996).

3.- G. Balme, E. Bossharth, N. Monteiro, Eur. J. Org. Chem. 21, 4101, (2003)

4.- J. Zhu, H. Bienayme, Multicomponent Reactions. Wiley-VCH: Weinheim, 2005.

5.- For a special issue on MCRs, see Tetrahedron 61, 11299 (2005).

6.- J.C. Menendez, Synthesis 15, 2624 (2006).

7.- M. Plunkett, J. A. Ellman, Sci. Am. 276, 68, (1997).

8.- L. F. Tietze, A. Modi, Med. Res. Rev. 20, 304, (2000).

9.- L. Weber, Drug Discovery Today 7, 143, (2002).

10.- H.P. Li, Pestic. Sci. Adm. 25, 28, (2004).

11.- K.T. Ren, Y.H. Li, H.Z. Yang, Chin. J. Pesticides 38, 1, (1999).

12.- J.M. Coret, US pat. 7,329,770, 2008.
13.- L. Jiang, H. Wang, M. Wang, X. Teng, Molecules 15, 1074, (2010).

14.- K.Y. Chen, C.T. Huang, Int. J. Appl. Sci. Eng. 2, 286, (2004).

15.- R. Schurter, H. Rempfler, US pat. 4,713,109, 1987.

16.- G. Seifert, A.R. Sting, B. Urwyler, US Pat. 6,175,018, 2001.

17.- C.D.S Tomlin, The pesticide manual, $13^{\text {th }}$ edition; published by BCPC, UK, 2003

18.- P. Zhong, H. Hu, S. Guo, Synth. Commun. 34, 4301, (2004).

19.- G. Blotny, Tetrahedron 62, 9507, (2006).

20.- H.L. Rayle, L. Fellmeth, Org. Process Res. Dev. 3, 172, (1999).

21.- B.P. Bandgar, S.S. Sawant, Synth. Commun. 36, 859, (2006).

22.- K. Venkataraman, D.R. Wagle, Tetrahedron Lett. 32, 3037, (1979).

23.- A. Mobinikhaledi,; N. Foroughifar, M.A. Bodaghi Fard, H. Moghanian, S. Ebrahimi, M. Kalhor, Synth. Commun. 39, 1166, (2009).

24.- A. Mobinikhaledi, N. Foroughifar, M. Kalhor, Syn. Reac. Inorg. Met-org. Chem. 39, 509, (2009). 